\title{
Educación matemática interdisciplinaria en la escuela - ejemplos y experiencias
}

\author{
Interdisciplinary Mathematicas Education at school - \\ examples and experiences.
}

\author{
Rita Borromeo Ferri \\ Universidad de Kassel, Instituto de Matemática \\ Pontificia Universidad Católica de Valparaíso \\ borromeo@mathematik.uni-kassel.de
}

\section{RESUMEN}

La Educación Matemática Interdisciplinaria tiene el objetivo de conectar las matemáticas con otras disciplinas. El beneficio para los estudiantes en la escuela es cuando reconocen que las matemáticas se pueden encontrar en muchas otras áreas de la vida, lo que es excelente para su visión de las matemáticas. El desafío para los maestros de todo el mundo sigue siendo cómo desarrollar y conducir clases de matemáticas interdisciplinarias. Al respecto, esta publicación brindará varios ejemplos, experiencias y resultados de estudios empíricos sobre este tema con un enfoque en las áreas STEM (en inglés Science, Technology, Engineering and Mathematics).

Palabras clave: educación matemática interdisciplinaria, educación STEM, educación docente para STEM. 


\begin{abstract}
Interdisciplinary Mathematical Education aims to connect mathematics with other disciplines. The benefit for students in school is, when they recognize that mathematics can be found in many other areas of life and disciplines, that they get another view of mathematics. The challenge for teachers around the world remains how to develop and conduct interdisciplinary math classes. In this regard, this publication will provide several examples, experiences and results of empirical studies on this topic with a focus on STEM areas.
\end{abstract}

Key words: Interdisciplinary Mathematics Education, STEM education, Teacher Education for STEM.

\title{
1. EDUCACIÓN INTERDISCIPLINARIA DE MATEMÁTICAS - CARACTERIZACIÓN Y SIGNIFICADO
}

La Educación Matemática Interdisciplinaria (EMI) se está discutiendo actualmente en todas partes. ¿Por qué este tema se percibe como significativo? ¿Qué caracteriza la educación matemática interdisciplinaria? ¿Cómo se aprende a enseñar esto? ¿Hay ejemplos concretos de clases?

Estas son muchas preguntas válidas. Aunque la EMI no es un tema nuevo a nivel mundial, ha ganado más importancia en los últimos años, sobre todo la educación STEM (en inglés Science, Technology, Engineering and Mathematics). En esta sección, se aclarará brevemente el concepto de Educación Matemática Interdisciplinaria. Luego, se darán argumentos sobre el por qué la EMI es importante para los estudiantes en la escuela.

\subsection{Concepto y conocimiento cientifico de educación matemática interdisciplinaria}

El conocimiento científico de la Educación Matemática Interdisciplinaria que considere tanto estudios prácticos como empíricos, es posible de ser destacado en dos publicaciones autorizadas en los últimos años: los libros de Williams et al. (2016) y Doig et al. (2019). Por un lado, se presentan diferentes enfoques del término EMI, siendo aclarado y explicado para plantear sugerencias para su implementación en el aula. Roth, coautor del libro de Williams et al. (2016) tiene una caracterización pragmática de EMI a la que me refiero: "La interdisciplinariedad denota el hecho, la calidad o la condición de dos o más campos 
académicos o ramas del aprendizaje. Los proyectos interdisciplinarios tienden a cruzar los límites tradicionales entre las disciplinas académicas" (Roth, 2014, p. 317).

Volveré a esa definición a medida que me acerque a la educación STEM y aclare mi propio enfoque de EMI para educadores y estudiantes. La revisión de la literatura muestra que la interdisciplinariedad en la educación matemática es un campo de investigación relativamente poco desarrollado. La literatura existente sugiere que a veces hay resultados beneficiosos del trabajo interdisciplinario en los planes de estudio integrados, que a menudo involucran proyectos. Estos resultados robustecen los resultados de aprendizaje motivacionales, afectivos y de resolución de problemas, y tal vez, una mejor comprensión de lo que es una disciplina, y cómo diferentes disciplinas pueden contribuir a una actividad útil (Williams et al., 2016).

\subsection{Significado de la Enseñanza Matemática Interdisciplinar}

Aquí hay tres razones claves por las cuales EMI puede ser significativa para los estudiantes. Para ello, los educadores también deben realizar EMI, ya que solo cuando los alumnos experimentan EMI puede tener éxito en diferentes niveles.

\section{a. Cambia la perspectiva de las matemáticas}

Las opiniones sobre las matemáticas generalmente se denominan "mathematical beliefs". Por ejemplo, Schoenfeld (1985, p.44) define las creencias como "one's own mathematical world" ("el propio mundo matemático"), aunque existen muchos otros resultados de investigaciones sobre "mathematical beliefs" (por ejemplo, Hannula et al., 2016). Sobre todo, se podría demostrar que las "mathematical beliefs" cambian durante un período de tiempo más largo a pesar de una cierta estabilidad. Si los alumnos solo conocen las matemáticas como aritmética obstinada y no ven que las matemáticas se usan y aplican también en otras disciplinas, entonces su punto de vista no cambia. A través del aprendizaje interdisciplinario, aunque solo sea a través de algunas actividades, los alumnos se dan cuenta de que las matemáticas son emocionantes y necesarias en la vida real. 


\section{b. Comprensión del contenido matemático por diferentes disciplinas}

Hay contenidos matemáticos que se necesitan en varias disciplinas y que a menudo no están en red. Un ejemplo es la fórmula de la Ley Espacio-Tiempo, que también es tratada en la física. Sin embargo, muchos alumnos no ven ninguna correlación si la fórmula se trata en las clases de matemáticas y luego en las de física. A medida que los maestros introducen esta fórmula de manera interdisciplinaria y combinan directamente las matemáticas con la física, los alumnos tienen la oportunidad de comprender mejor el contenido. Además, los experimentos prácticos realizados en física facilitan que los alumnos cuestionen y "traduzcan" el formato de la fórmula.

\section{c. Redes de áreas de conocimiento}

Este aspecto se relaciona fuertemente con el punto anterior. Dependiendo de la calificación, los alumnos tienen diferentes asignaturas en el horario. Las matemáticas son una materia escolar que los alumnos deben probar constantemente desde la escuela primaria hasta el final de los estudios secundarios. Pero para muchos estudiantes, las matemáticas son solo una "materia escolar" y no una disciplina (Imagen 1). Lo mismo se aplica, por ejemplo, al español, la historia natural o la química. En todas las materias, los alumnos adquieren competencias y conocimientos, que pueden conectarse en red a través del aprendizaje interdisciplinario. Para este propósito, los límites entre las materias escolares deben superarse, de modo que los alumnos reconozcan las conexiones. Esta es la tarea de los profesores, ya que demanda un trabajo interdisciplinario.

\section{ENSEÑAR EMI - Y EL "CROSS LINK TEACHING APROACH"}

Enseñar EMI se transforma en uno de los mayores desafíos cuando es reconocida su ausencia en la formación de profesores. La idea del EMI, previamente mencionado, no es nueva, pero su promoción inició hace mucho tiempo. Al respecto, Klafki (1998) describe las ventajas con respecto a la comprensión del contenido de los estudiantes desde diferentes perspectivas, siendo evidente a través de experimentos de enseñanza con varias disciplinas. A menudo, cuando se habla de EMI, se ha convertido en sinónimo de la educación en Ciencia, Tecnología, Ingeniería y Matemáticas (en inglés, STEM) se ha convertido en sinónimo de esto. La EMI no está, o no debería estar, limitado al "STE", y podría incluir otras disciplinas en todo el plan de estudios. A menudo depende del conocimiento y las preferencias del maestro para que una se- 
gunda disciplina cruce los "límites tradicionales", ya sea en las Artes, como en STE(A)M, o en las humanidades o el deporte. En Alemania, por ejemplo, los maestros de secundaria tienen que estudiar dos materias escolares en la universidad para ser empleados como profesores de escuela más adelante, iy todas las combinaciones posibles de materias están permitidas! Teniendo en cuenta la discusión actual, el objetivo es tener profesores calificados en educación STEM. ¿Qué esperamos de ellos que sepan? ¿Deberían ser expertos en los cuatro campos STEM?

Esta pregunta fue el punto de partida para mi conceptualización teórica de STEM desde una perspectiva docente y educativa, que también se basa en el debate existente en la educación STEM: distingo entre el "singlefield teaching approach" y el "cross-link field teaching approach". El primer enfoque describe la promoción muy profundamente de un solo campo STEM en la escuela, por ejemplo, un entorno de aprendizaje de ingeniería (como un ejemplo para esto, ver English \& King, 2015). El segundo se caracteriza de la siguiente manera: se puede hablar de "cross-link", si se combinan al menos dos disciplinas (científicas) durante una lección o dentro de la unidad de lección completa y se reflejan con los estudiantes en un nivel metacognitivo. Esta definición también fue el resultado de un entorno de aprendizaje con el tema "Leonardo-da-Vinci-Bridge" (ver la siguiente sección), que fue desarrollado por mí y mi colega de Matemática Aplicada, Ingeniería Mecánica, Física y Arte.

\subsection{Ejemplos y experiencias}

En esta sección se dan dos ejemplos para EMI, ambos para la escuela secundaria. Dentro del primer ejemplo, se desarrolló una unidad de lección y se realizó en el Grado 10 (15 años aproximadamente). Allí se enseñaron matemáticas y deportes interdisciplinarios. En el segundo ejemplo, se creó una unidad de lección con el tema "Leonardo-da-Vinci", pero aquí informo específicamente sobre el tema "Leonardo-Bridge". En ambos estudios se investigó cómo es posible incrementar las "mathematical beliefs" en los alumnos, como se mencionó anteriormente. Por ello, se utilizó un cuestionario para los alumnos antes y después de la unidad de enseñanza. Además, se investigó si los alumnos podían explicitar su entendimiento sobre cómo estaban conectadas las disciplinas. Los dos ejemplos dan una idea cualitativa sobre cómo se puede realizar la EMI, por ello se determina una pequeña muestra. Debido al límite de espacio en este reporte, solo se puede realizar una breve presentación de ambos casos. 


\section{Matemáticas y Deportes (secundaria, décimo grado)}

Junto con mi grupo de investigación decidimos combinar estas dos disciplinas, porque era un área hasta ahora poco explorada. Ludwig y Reit (2013) señalan que el deporte posee un gran estatus entre los jóvenes. La motivación de los alumnos obtenidos en el deporte a través de la experiencia física también se puede utilizar para las lecciones de matemáticas. Los contenidos se conectan entre sí y una referencia se vuelve transparente para los alumnos. El estudio se realizó con 13 estudiantes de décimo grado y fue impartido por un maestro experimentado. La serie de clases consistió en un total de seis clases dobles (6x90 min). De estos, cuatro clases dobles se atribuyeron a las clases de matemáticas y dos clases dobles a la educación física. La tarea básica era: "¿Es el ángulo de tiro el ángulo óptimo para un tiro libre en baloncesto?" Además, los estudiantes recibieron un texto en el que se mostraba la Imagen 1:

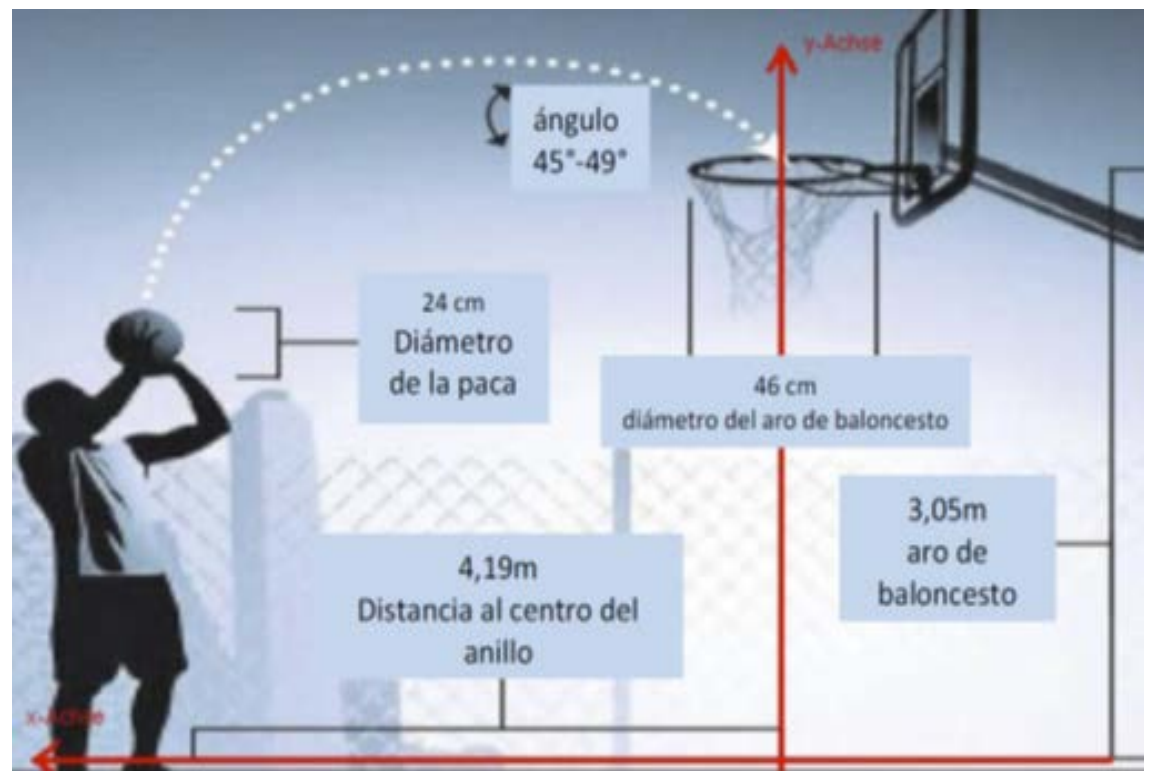

Imagen 1. Descripción de la información en la combinación Matemáticas y Deportes. Fuente: elaboración propia de la autora. 
El contenido propuesto en la combinación disciplinaria era sobre funciones cuadráticas. Esta tarea requiere que los estudiantes usen las matemáticas de manera flexible y de varias maneras. Específicamente, el contenido de cada clase se puede ver en la tabla 1. Dentro de la serie de clases se utilizaron diferentes métodos de enseñanza. Los estudiantes también trabajaron con el software de geometría dinámica Geogebra.

Tabla 1

Planificación de las clases en Matemáticas y Deportes.

\begin{tabular}{|c|c|c|}
\hline Clases & Tema & Objetivo de aprendizaje \\
\hline $\begin{array}{l}\text { Primera } \\
\text { clase } \\
\text { doble }\end{array}$ & $\begin{array}{l}\text { Análisis del artículo periodís- } \\
\text { tico sobre el diseño del ba- } \\
\text { loncesto desde un aspecto } \\
\text { matemático. Desarrollo de } \\
\text { estrategias de resolución de } \\
\text { problemas. }\end{array}$ & $\begin{array}{l}\text { Los alumnos desarrollan la capacidad } \\
\text { de matematizar la situación real y la } \\
\text { competencia del pensamiento en la re- } \\
\text { solución de problemas. }\end{array}$ \\
\hline $\begin{array}{l}\text { Segunda } \\
\text { clase } \\
\text { doble }\end{array}$ & $\begin{array}{l}\text { Los estudiantes identifican } \\
\text { tasas de éxito. Ellos crean se- } \\
\text { cuencias de video de tiros li- } \\
\text { bres con diferentes ángulos } \\
\text { de expulsión. }\end{array}$ & $\begin{array}{l}\text { Los alumnos expanden la capacidad de } \\
\text { lanzamiento. Ellos enlazan tres expe- } \\
\text { riencias prácticas con diferentes tipos } \\
\text { de conocimiento teórico }\end{array}$ \\
\hline $\begin{array}{l}\text { Tercera } \\
\text { clase } \\
\text { doble }\end{array}$ & $\begin{array}{l}\text { Configurar ecuaciones con } \\
\text { tres variables dado tres pun- } \\
\text { tos para la reconstrucción de } \\
\text { parábolas. }\end{array}$ & $\begin{array}{l}\text { Los alumnos aprenden a construir la } \\
\text { ecuación de una función racional de } \\
\text { grado dos dado tres puntos. }\end{array}$ \\
\hline $\begin{array}{l}\text { Cuarta } \\
\text { clase } \\
\text { doble }\end{array}$ & $\begin{array}{l}\text { La reconstrucción de la fun- } \\
\text { ción parabólica dado tres } \\
\text { puntos y la provisión de un } \\
\text { ángulo de tiro. }\end{array}$ & $\begin{array}{l}\text { Los alumnos aprenden a obtener tres } \\
\text { puntos del material de video. Ellos re- } \\
\text { construyen la función parabólica del } \\
\text { video, entonces, los estudiantes apren- } \\
\text { den a leer el ángulo de tiro, interpretary } \\
\text { clasificar el resultado. }\end{array}$ \\
\hline $\begin{array}{l}\text { Quinta } \\
\text { clase } \\
\text { doble }\end{array}$ & $\begin{array}{l}\text { Los alumnos determinan las } \\
\text { tasas de éxito para tiros libres } \\
\text { adaptados. }\end{array}$ & $\begin{array}{l}\text { Los alumnos expanden el experimento } \\
\text { "Tiros en el Baloncesto" y reconocen la } \\
\text { conexión entre los diferentes tipos de } \\
\text { tiros y tasas de éxito. }\end{array}$ \\
\hline $\begin{array}{l}\text { Sexta } \\
\text { clase } \\
\text { doble }\end{array}$ & $\begin{array}{l}\text { Comparación de tasas de éxi- } \\
\text { to. Reflejo de la serie (o planifi- } \\
\text { cación) de clases. Prueba. }\end{array}$ & $\begin{array}{l}\text { Los alumnos aprenden a obtener resul- } \\
\text { tados y su interpretación. Los alumnos } \\
\text { aprenden a obtener resultados e inter- } \\
\text { pretar individualidades al reflejo de un } \\
\text { sujeto. }\end{array}$ \\
\hline
\end{tabular}

Fuente: elaboración propia de la autora. 
En términos de control de aprendizaje y prueba posterior, era importante interrogar y conectar las competencias con ambas disciplinas, por ello, el enfoque estaba en los objetivos de aprendizaje matemático. Se estableció un sistema lineal de ecuaciones, se resolvió y determinó el ángulo de inclinación de una parábola en un punto fijo. Por otro lado, se consultó sobre el conocimiento de los alumnos respecto a las reglas del baloncesto. Finalmente, en una tarea, se combinaron las dos disciplinas, matemáticas y deporte, y se probó el conocimiento. Los resultados mostraron que los estudiantes reconocieron las relaciones de las materias. Para los alumnos, aún no era familiar que una prueba involucrara dos disciplinas, ya que no es común este caso, lo que implicó el cambio de sus "mathematical beliefs". Si antes de la serie de clases los alumnos estaban más interesados en visualizar las matemáticas como un conjunto rígido de reglas, después de la unidad de enseñanza dejaron en primer plano el aspecto presentado. El contenido del área temática dentro del entorno de los alumnos fue fundamental para el éxito de la serie de clases. Además, incluir información de relevancia de la vida de los alumnos podría evocar un contenido significativo de la serie de clases.

\section{Matemáticas, Física, Arte, Ingeniería - Puente Leonardo-da-Vinci (secundaria, noveno grado)}

Con el objetivo de enseñar matemáticas, ingeniería, física y arte interdisciplinaria, se propone un eficaz trabajo colaborativo con personas de varias áreas. Surge el nombre de Leonardo da Vinci como un gran universalista, focalizando en el clásico Puente Leonardo el objetivo del trabajo interdisciplinario. Los alumnos comprenderán las fuerzas que actúan sobre las superficies de contacto de las barras y, en consecuencia, se pueden imaginar pares de fuerzas y los momentos resultantes que actúan sobre las barras. En base a esta información, los estudiantes comprenderán las propiedades de carga general de los puentes y los diseños típicos de puentes como puentes suspendidos de celosía, vigas, arcos y cables. Un grupo de expertos compuesto por educación matemática, matemática aplicada, ingeniería civil y un artista de esculturas creó un ambiente de aprendizaje común, que puede adaptarse de acuerdo con las habilidades de los estudiantes de nivel secundario (noveno a doceavo grado).

El enfoque "Inquiry-based learning (IBL)" (Edelson et al. 1999) construye la base para el módulo de aprendizaje, ya que se adapta perfectamente a los requisitos de aprendizaje de STEM. El enfoque IBL incorpora un proceso para que los estudiantes exploren el mundo, lo que lleva a hacer preguntas, 
hacer descubrimientos y realizar experimentos en la búsqueda de nuevos entendimientos. Además, el IBL valora las ideas y preguntas del alumno para trabajar hacia el logro de los resultados deseados, con el conocido procedimiento / ciclo:

Preguntar $\rightarrow$ Investigar $\rightarrow$ Crear $\rightarrow$ Discutir $\rightarrow$ Reflexionar $\rightarrow$ Preguntar ...

El módulo de enseñanza está desarrollado para 6 lecciones con 90 minutos cada una.

Las preguntas de investigación fueron similares al ejemplo anterior:

1. ¿Qué percepción del enfoque de enlaces cruzados se puede reconstruir con los estudiantes después de la unidad de clases?

2. ¿Las creencias matemáticas de los estudiantes cambian después de la unidad de clases?

La muestra comprendió 28 estudiantes de un grado 9. La unidad de clase de 6 horas comprendió tres fases:

Fase A: Antecedentes históricos e introducción a Leonardo da Vinci/ Modelado del puente Leonardo;

Fase B: Evaluación de contenido del puente (matemáticas, física, ingeniería, arte);

Fase C: Resumen de los hallazgos y reflexión. Para obtener una visión más profunda del contenido de la unidad de enseñanza, la Fase A se describirá con más detalle. 


\section{Puente Leonardo}

Contenido clases 1 y 2

El maestro presenta una foto del puente Leonardo. Discusión con los alumnos sobre Leonardo da Vinci y sus talentos (desarrollo de un mapa mental con los alumnos).

El maestro presenta el objetivo de la unidad: "Ahora ustedes son investigadores y prepararán un libro de investigación para una documentación sistemática sobre sus hallazgos sobre el puente Leonardo. Al final, los libros de investigación también se presentan como una obra de arte".

Los estudiantes trabajan en parejas para descubrir el fenómeno, por qué el puente funciona sin pegamento ni clavos. Ellos escriben sus ideas.

Los estudiantes intentan construir el puente sobre la base de la foto y desarrollan preguntas de investigación:

-¿Quién construye un puente con el mayor alcance?

- ¿Quién construye un puente con el mínimo de duelas?

Los estudiantes realizan trabajos experimentales y finalmente presentan sus ideas en el pleno. La reflexión comienza con un enfoque a la realidad. Los estudiantes aprenden sobre el efecto de autobloqueo.

A continuación, se puede ver la última parte de la fase C de la unidad de clases, lo que significa la reflexión sobre el contenido y la transferencia del hallazgo de Leonardo da Vinci al presente.

\section{Última parte de la clase 6}

Los libros de investigación de los alumnos se exponen en el aula. Antes de que los estudiantes y el maestro discutan cómo debe ser un buen libro de investigación, cada libro fue calificado por los estudiantes. El libro y, por lo tanto, el estudiante con más puntos fue premiado.

Última discusión plenaria: se muestra una foto del puente Leonardo en As en Oslo.

Evaluación de todo el proyecto: los estudiantes escriben comentarios sobre tarjetas de diferentes colores. 
IBL como estilo de enseñanza fue una experiencia completamente nueva para los estudiantes, ya que les requirió tiempo para comprender que tenían que actuar de una manera más independiente, que no estaba centrada en el maestro. Los alumnos quedaron fascinados al explorar y construir el puente Leonardo durante el trabajo grupal, para aprender y comprender las matemáticas, la física, la ingeniería y los antecedentes artísticos.

Al mostrar a los estudiantes el puente en Oslo, por ejemplo, un estudiante dijo: "El puente se ve muy bien y la estática del puente depende de cómo se realiza la conexión matemática y física correctamente". Fue fantástico observar el conocimiento de los estudiantes, porque el objetivo no era solo construir el puente Leonardo y divertirse, sino mostrarles o hacerlos pensar en las matemáticas, la física y el arte detrás de él. Para nosotros, este fue un gran éxito en el nivel de observación.

Como ya se mencionó, una pregunta abierta en la prueba previa y posterior para los estudiantes fue estudiar qué conexión ven o no ven entre las matemáticas y otras disciplinas. No todos los estudiantes respondieron esta pregunta, exactamente 18 de 28 , sin embargo, uno podría reconstruir el enlace cruzado después de la unidad de clases. Las siguientes declaraciones son de Karen (15 años). Antes y después de la unidad de clases, ella escribió:

Declaración antes de la unidad de clase:

"No veo una conexión entre las matemáticas y otras materias, excepto la química".

Declaración después de la unidad de lección:

"Las matemáticas son la base de la ciencia. Las lecciones me mostraron cómo las matemáticas están vinculadas con el arte, la física y la arquitectura ".

La mayoría de los estudiantes no estaban segurossobre qué otras disciplinas se podrían vincular con las matemáticas. La ciencia a menudo estaba en el foco, pero ni un solo estudiante mencionó, por ejemplo, arte, ingeniería o IT (Information Technology). Después de la unidad de clase, Karen estaba más sensibilizada. En la declaración de Karen, su "nueva" conexión notada se hace evidente, porque se refiere al proyecto Leonardo, cuando mencionó "arte, física y arquitectura". El análisis mostró más declaraciones similares sobre el vínculo cruzado de los alumnos. Los resultados de las creencias matemáticas de los estudiantes son interesantes. El resultado central es que se podría establecer un cambio en las "mathematical beliefs" de los alumnos, aunque 
la intervención fue breve, pero aparentemente efectiva en la forma en que los estudiantes pensaban acerca de las matemáticas antes y después de la unidad de clase.

En la enseñanza diaria no siempre es posible utilizar IBL, pero es de gran ayuda para tener una idea de cómo las matemáticas se pueden "crosslinked" con otros campos y también que las matemáticas se notan como una disciplina de investigación.

\section{DISCUSIÓN}

El objetivo del artículo fue, por un lado, aclarar el término de la Educación Matemática Interdisciplinaria (EMI). Por otro lado, presentar algunos ejemplos y experiencias sobre cómo se puede enseñar EMI en la escuela. Además, el término del "cross-link teaching approach" se mostró concretamente con dos ejemplos. Cuando se enseña EMI, es importante que los alumnos también entiendan a nivel metacognitivo, que dos o más disciplinas están vinculadas. La responsabilidad del maestro es hacer que los alumnos tengan una conexión explícita entre la matemática y otras materias. Los ejemplos aclararon que en una unidad de enseñanza se pueden conectar varias disciplinas y se puede cambiar fácilmente el nivel de las matemáticas. Al mirar la unidad de enseñanza de Puente Leonardo, puede enfocarse en temas difíciles en matemáticas y física o puede hacer que sea más fácil para los estudiantes en la escuela primaria. Los ejemplos también dejaron en claro que, para implementar EMI, los maestros deben ser educados y entrenados para ello. Hasta ahora, no hay o solo unos pocos cursos en todo el mundo, que se ofrecen específicamente para EMI. Esta es la razón por la cual EMI rara vez está en la escuela. Por lo tanto, tenemos que hacer más en la formación de profesores para que los alumnos de la escuela finalmente se beneficien de EMI.

\section{REFERENCIAS BIBLIOGRÁFICAS}

Doig, B., Williams, J., Swanson, D., Borromeo Ferri, R. \& Drake, P. (Ed.). (2019). Interdisciplinary Mathematics Education - The State of the Art and Beyond. New York: Springer.

Edelson, D., Gordin, D. \& Pea, R. (1999). Addressing the Challenges of InquiryBased Learning Through Technology and Curriculum Design. The Journal of the Learning Sciences, 8(3-4), 391-450. DOI: https://doi.org/ 10.1080/10508406.1999.9672075 
English, L. \& King, D. (2015). STEM learning through engineering design: fourth-grade students' investigations in aerospace. The International Journal of STEM Education, 2, 2-18. Recuperado de https://stemeducationjournal.springeropen.com/articles/10.1186/s40594-015-0027-7

Hannula, M.S., Di Martino, P., Pantziara, M., Zhang, Q., Morselli, F., Heyd-Metzuyanim, E., Lutovac, S., Kaasila, R., Middleton, J.A., Jansen, A., \& Goldin, G.A. (2016). Attitudes, Beliefs, Motivation and Identity in Mathematics Education. An Overview of the Field and Future Directions. New York: Springer.

Klafki, W. (1998). Schlüsselprobleme der modernen Welt und die Aufgaben der Schule - Grundlinien einer neuen Allgemeinbildungskonzeption in internationaler/interkultureller Perspektive. In: Gogolin, I., KrügerPotratz, M., Meyer, M.A. (eds.). Pluralität und Bildung. Schriften der Deutschen Gesellschaft für Erziehungswissenschaften (DGfE) (pp. 95-123). Wiesbaden: VS Verlag für Sozialwissenschaften. DOI: https:// doi.org/10.1007/978-3-663-11056-9_14

Ludwig, M. \& Reit, X.-R. (2013). Eine empirische Studie zum mathematischen Modellieren im Sport. In R. Borromeo Ferri, G. Greefrath, \& G. Kaiser (Hrsg.), Realitätsbezüge im Mathematikunterricht: Mathematisches Modellieren für Schule und Hochschule, (pp. 95-123). Wiesbaden: springer Spektrum.

Roth, W.M. (2014). Interdisciplinary approaches in mathematics education. In S. Lerman (Ed.), Encyclopedia of mathematics education (pp. 647650). Berlin, Heidelberg: Springer.

Schoenfeld, A. (1985). Explorations of Students' Mathematical Beliefs and Behavior. Journal for Research in Mathematics Education, 20(4), 338355. DOI: $10.2307 / 749440$

Williams, J., Roth, W.M., Swanson, D., Doig, B., Groves, S., Omuvwie, M., Borromeo Ferri, R., \& Mousoulides, N. (2016). Interdisciplinary Mathematics Education. A State of the Art. New York: Springer. 